九州大学学術情報リポジトリ

Kyushu University Institutional Repository

\title{
ALL-PAIRWISE MULTIPLE COMPARISON FOR NORMAL VARIANCES
}

Imada, Tsunehisa

Department of Management, Tokai University

https://doi.org/10.5109/2233855

出版情報: Bulletin of informatics and cybernetics. 50, pp.19-26，2018-12. Research Association of Statistical Sciences

バージョン：

権利関係 : 
ALL-PAIRWISE MULTIPLE COMPARISON FOR NORMAL VARIANCES

by

Tsunehisa IMADA

Reprinted from the Bulletin of Informatics and Cybernetics Research Association of Statistical Sciences, Vol.50

FUKUOKA, JAPAN

2018 


\title{
ALL-PAIRWISE MULTIPLE COMPARISON FOR NORMAL VARIANCES
}

\author{
By
}

Tsunehisa IMADA*

\begin{abstract}
In this study we discuss the all-pairwise multiple comparison for checking differences among normal variances. Although Imada (2018) determined two kinds of conservative critical values for pairwise comparison for a specified significance level using Bonferroni's inequality and the improved Bonferroni's inequality respectively, we discuss how to determine the critical value for pairwise comparison satisfying a specified significance level exactly. Finally, we give some numerical results regarding critical values and power of the test intended to compare the exact critical value and the conservative critical values.
\end{abstract}

Key Words and Phrases: Iterated integration, Power of the test, Single step procedure.

\section{Introduction}

Assume there are independent normal random variables $X_{1}, X_{2}, \ldots, X_{K}$ and $X_{k}$ is distributed according to normal $N\left(\mu_{k}, \sigma_{k}^{2}\right)$ for $k=1,2, \ldots, K$. For testing whether $\mu_{1}=\mu_{2}=\cdots=\mu_{K}$ or not by the analysis of variance the assumption $\sigma_{1}^{2}=\sigma_{2}^{2}=$ $\cdots=\sigma_{K}^{2}$ is necessary. The assumption is also necessary for multiple comparison procedures proposed by Dunnett (1955) and Tukey (1953) for checking specific differences among $\mu_{1}, \mu_{2}, \ldots, \mu_{K}$. When the hypothesis $\sigma_{1}^{2}=\sigma_{2}^{2}=\cdots=\sigma_{K}^{2}$ is rejected, we occasionally want to find the pair $\sigma_{i}^{2}, \sigma_{j}^{2}$ satisfying $\sigma_{i}^{2} \neq \sigma_{j}^{2}$. Imada (2018) discussed the multiple comparison with a control for comparing $\sigma_{1}^{2}$ with $\sigma_{2}^{2}, \sigma_{3}^{2}, \ldots, \sigma_{K}^{2}$ simultaneously and the all-pairwise multiple comparison for $\sigma_{1}^{2}, \sigma_{2}^{2}, \ldots, \sigma_{K}^{2}$ (cf. Dunnett (1955) and Tukey (1953)) . For the multiple comparison with a control Imada (2018) determined the critical value for pairwise comparison satisfying a specified significance level exactly and formulated the power of the test under a specified alternative hypothesis. For the all-pairwise multiple comparison Imada (2018) determined two kinds of conservative critical values for pairwise comparison for a specified significance level using Bonferroni's inequality and the improved Bonferroni's inequality respectively and calculated the power of the test by Monte Carlo simulation. The aim of this study is to determine the critical value for pairwise comparison of the all-pairwise multiple comparison satisfying a specified significance level exactly. We give some numerical results regarding critical values and power of the test intended to compare the exact critical value with the conservative critical values.

\footnotetext{
* Department of Management, Tokai University, 9-1-1 Toroku Higashi-ku Kumamoto, Japan 862-8652 Japan. tel +81-96-386-2731, timada@ktmail.tokai-u.jp
} 


\section{All-pairwise multiple comparison for normal variances}

We consider the all-pairwise multiple comparison for $\sigma_{1}^{2}, \sigma_{2}^{2}, \ldots, \sigma_{K}^{2}$ using a sample $x_{k 1}, x_{k 2}, \ldots, x_{k n_{k}}$ from $N\left(\mu_{k}, \sigma_{k}^{2}\right)$ for $k=1,2, \ldots, K$. Intended to compare $\sigma_{k}^{2}$ and $\sigma_{l}^{2}$ for $1 \leq k<l \leq K$ we set up a null hypothesis and its alternative hypothesis as

$$
H_{k, l}: \sigma_{k}^{2}=\sigma_{l}^{2} \quad \text { vs. } H_{k . l}^{A}: \sigma_{k}^{2} \neq \sigma_{l}^{2}
$$

and consider the simultaneous test of all $H_{k, l}$ s. Letting

$$
\nu_{k}^{2}=\frac{\sum_{i=1}^{n_{k}}\left(x_{k i}-\bar{x}_{k}\right)^{2}}{n_{k}-1}
$$

for $k=1,2, \ldots, K$, we use the statistic

$$
F_{k, l}=\frac{\nu_{l}^{2}}{\nu_{k}^{2}}
$$

for testing $H_{k, l}$. If $n_{1}, n_{2}, \ldots, n_{K}$ are unbalanced, it is preferable to set up appropriate critical values for each $H_{k, l}$. However, we set up common critical values for all $H_{k, l}$ s for simplicity. We specify a lower critical value $c_{1}$ and an upper critical value $c_{2}$ satisfying $0<c_{1}<c_{2}$. If $F_{k, l}<c_{1}$ or $c_{2}<F_{k, l}$, we reject $H_{k, l}$. Otherwise, we retain $H_{k, l}$. Since

$$
F_{k, l}=\frac{\nu_{l}^{2}}{\nu_{k}^{2}}<c_{1} \Leftrightarrow c_{1}^{-1}<F_{k, l}^{-1}=\frac{\nu_{k}^{2}}{\nu_{l}^{2}}
$$

and

$$
c_{2}<F_{k, l}=\frac{\nu_{l}^{2}}{\nu_{k}^{2}} \Leftrightarrow F_{k, l}^{-1}=\frac{\nu_{k}^{2}}{\nu_{l}^{2}}<c_{2}^{-1},
$$

we restrict $c_{1}, c_{2}$ as

$$
c_{2}=c_{1}^{-1}=c>1 \text {. }
$$

Then, we obtain

$$
c^{-1}<F_{k, l}=\frac{\nu_{l}}{\nu_{k}}<c \Leftrightarrow c^{-1}<F_{k, l}^{-1}=\frac{\nu_{k}}{\nu_{l}}<c
$$

for all $(k, l)$ s satisfying $1 \leq k<l \leq K$. The probability that at least one hypothesis among $H_{k, l}$ s is rejected is

$$
P\left(\min _{1 \leq k<l \leq K} F_{k, l}<c^{-1} \text { or } c<\max _{1 \leq k<l \leq K} F_{k, l}\right) .
$$

We want to determine $c$ so that

$$
P\left(\min _{1 \leq k<l \leq K} F_{k, l}<c^{-1} \text { or } c<\max _{1 \leq k<l \leq K} F_{k, l}\right)=\alpha
$$

for a specified significance level $\alpha$ under the assumption that $\sigma_{1}^{2}=\sigma_{2}^{2}=\cdots=\sigma_{K}^{2}$. 


\subsection{Conservative critical values}

Imada (2018) determined two kinds of conservative critical values using Bonferroni's inequality and the improved Bonferroni's inequality respectively. By Bonferroni's inequality we obtain

$$
P\left(\min _{1 \leq k<l \leq K} F_{k, l}<c^{-1} \text { or } c<\max _{1 \leq k<l \leq K} F_{k, l}\right) \leq \sum_{1 \leq k<l \leq K} P\left(F_{k, l}<c^{-1} \text { or } c<F_{k, l}\right) .
$$

Letting $f_{k, l}(x)$ be the probability density function of $F$-distribution with degrees of freedom $\left(n_{l}-1, n_{k}-1\right)$, we obtain

$$
\sum_{1 \leq k<l \leq K} P\left(F_{k, l}<c^{-1} \text { or } c<F_{k, l}\right)=\sum_{1 \leq k<l \leq K}\left\{1-\int_{c^{-1}}^{c} f_{k, l}(x) d x\right\} .
$$

If we determine $c$ so that

$$
\sum_{1 \leq k<l \leq K}\left\{1-\int_{c^{-1}}^{c} f_{k, l}(x) d x\right\}=\alpha,
$$

we obtain the conservative critical value by (3).

Next, by the improved Bonferroni's inequality we obtain

$$
\begin{gathered}
P\left(\min _{1 \leq k<l \leq K} F_{k, l}<c^{-1} \text { or } c<\max _{1 \leq k<l \leq K} F_{k, l}\right) \\
\leq \sum_{1 \leq k<l \leq K} P\left(F_{k, l}<c^{-1} \text { or } c<F_{k, l}\right) \\
-\sum_{l=1}^{K-2} \sum_{k=1}^{K-l-1} P\left(F_{k, k+l}<c^{-1} \text { or } c<F_{k, k+l}, F_{k+1, k+l+1}<c^{-1} \text { or } c<F_{k+1, k+l+1}\right) \\
-\sum_{l=1}^{K-2} P\left(F_{K-l, K}<c^{-1} \text { or } c_{2}<F_{K-l, K}, F_{1, l+2}<c^{-1} \text { or } c<F_{1, l+2}\right) .
\end{gathered}
$$

Let

$$
\lambda_{k_{1}, k_{2}}=\frac{n_{k_{2}}-1}{n_{k_{1}}-1}
$$

for each pair $\left(k_{1}, k_{2}\right)$ chosen from $1,2, \ldots, K$ and $g_{k}(x)$ be the probability density function of $\chi^{2}$-distribution with degrees of freedom $n_{k}-1$ for $k=1,2, \ldots, K$. Imada (2018) gave the formulation of

$$
P\left(F_{k, k+l}<c^{-1} \text { or } c<F_{k, k+l}, F_{k+1, k+l+1}<c^{-1} \text { or } c<F_{k+1, k+l+1}\right)
$$

for $k \geq 1, l \geq 1, k+l \leq K-1$ as follows. If $l=1$,

$$
\begin{gathered}
P\left(F_{k, k+1}<c^{-1} \text { or } c<F_{k, k+1}, F_{k+1, k+2}<c^{-1} \text { or } c<F_{k+1, k+2}\right) \\
=\int_{0}^{\infty} g_{k+1}\left(x_{k+1}\right)\left\{1-\int_{c^{-1} \lambda_{k, k+1}^{-1} x_{k+1}}^{c \lambda_{k, k+1}^{-1} x_{k+1}} g_{k}\left(x_{k}\right) d x_{k}\right\}\left\{1-\int_{c^{-1} \lambda_{k+1, k+2} x_{k+1}}^{c \lambda_{k+1, k+2} x_{k+1}} g_{k+2}\left(x_{k+2}\right) d x_{k+2}\right\} d x_{k+1} .
\end{gathered}
$$

If $l \geq 2$,

$$
P\left(F_{k, k+l}<c^{-1} \text { or } c<F_{k, k+l}, F_{k+1, k+l+1}<c^{-1} \text { or } c<F_{k+1, k+l+1}\right)
$$




$$
=\left\{1-\int_{c^{-1}}^{c} f_{k, k+l}(x) d x\right\}\left\{1-\int_{c^{-1}}^{c} f_{k+1, k+l+1}(x) d x\right\} .
$$

Furthermore, Imada (2018) gave the formulation of

$$
P\left(F_{K-l, K}<c^{-1} \text { or } c<F_{K-l, K}, F_{1, l+2}<c^{-1} \text { or } c<F_{1, l+2}\right)
$$

for $1 \leq l \leq K-2$ as follows. If $l=K-2$,

$$
\begin{gathered}
P\left(F_{2, K}<c^{-1} \text { or } c<F_{2, K}, F_{1, K}<c^{-1} \text { or } c<F_{1, K}\right) \\
=\int_{0}^{\infty} g_{K}\left(x_{K}\right)\left\{1-\int_{c^{-1} \lambda_{2, K}^{-1} x_{K}^{-1} x_{K}}^{c} g_{2}\left(x_{2}\right) d x_{2}\right\}\left\{1-\int_{c^{-1} \lambda_{1, K}^{-1} x_{K}}^{c \lambda_{1, K}^{-1} x_{K}} g_{1}\left(x_{1}\right) d x_{1}\right\} d x_{K} .
\end{gathered}
$$

If $K$ is even and $l=K / 2-1$,

$$
\begin{gathered}
P\left(F_{K / 2+1, K}<c^{-1} \text { or } c<F_{K / 2+1, K}, F_{1, K / 2+1}<c^{-1} \text { or } c<F_{1, K / 2+1}\right) \\
=\int_{0}^{\infty} g_{K / 2+1}\left(x_{K / 2+1}\right)\left\{1-\int_{c^{-1} \lambda_{K / 2+1, K} x_{K / 2+1}}^{c \lambda_{K / 2+1, K} x_{K / 2+1}} g_{k}\left(x_{K}\right) d x_{K}\right\} \\
\times\left\{1-\int_{c^{-1} \lambda_{1, K / 2+1}^{-1} x_{K / 2+1}}^{c \lambda_{1, K / 2+1}^{-1} x_{K / 2+1}} g_{1}\left(x_{1}\right) d x_{1}\right\} d x_{K / 2+1} .
\end{gathered}
$$

In other cases

$$
\begin{gathered}
P\left(F_{K-l, K}<c^{-1} \text { or } c<F_{K-l, K}, F_{1, l+2}<c^{-1} \text { or } c<F_{1, l+2}\right) \\
=\left\{1-\int_{c^{-1}}^{c} f_{K-l, K}(x) d x\right\}\left\{1-\int_{c^{-1}}^{c} f_{1, l+2}(x) d x\right\} .
\end{gathered}
$$

Therefore, we obtain the formulation of the right hand of (4). If we determine $c$ so that the right hand of (4) may be equal to $\alpha$, we obtain the less conservative critical value compared to the critical value determined by Bonferroni's inequality.

\subsection{Determination of the exact critical value}

We determine the critical value $c$ satisfying (2) exactly. (2) is equivalent to

$$
P\left(c^{-1}<F_{k, l}<c \text { for each pair }(k, l) \text { satisfying } 1 \leq k<l \leq K\right)=1-\alpha .
$$

We formulate $P\left(c^{-1}<F_{k, l}<c\right.$ for each pair $(k, l)$ satisfying $\left.1 \leq k<l \leq K\right)$. By (1) we obtain

$$
\begin{gathered}
c^{-1}<F_{k, l}<c \text { for each pair }(k, l) \text { satisfying } 1 \leq k<l \leq K \\
\Leftrightarrow \quad c^{-1} \nu_{l}<\nu_{k}<c \nu_{l} \text { for each pair }(k, l) \text { chosen from } 1,2, \ldots, K \\
\Leftrightarrow \quad c^{-1} \max \left\{\nu_{1}, \nu_{2}, \ldots, \nu_{K}\right\}<\nu_{k}<c \min \left\{\nu_{1}, \nu_{2}, \ldots, \nu_{K}\right\} \text { for } k=1,2, \ldots, K .
\end{gathered}
$$

The event defined by (5) and

$$
\min \left\{\nu_{1}, \nu_{2}, \ldots, \nu_{K}\right\}=\nu_{1}, \quad \max \left\{\nu_{1}, \nu_{2}, \ldots, \nu_{K}\right\}=\nu_{2}
$$


is equal to the event defined by

$$
\nu_{1} \leq \nu_{k} \leq \nu_{2}, c^{-1} \nu_{2}<\nu_{k}<c \nu_{1} \text { for } k=1,2, \ldots, K
$$

(6) is equivalent to

$$
\nu_{1} \leq \nu_{2} \leq c \nu_{1}, \quad \nu_{1}=\max \left\{c^{-1} \nu_{2}, \nu_{1}\right\}<\nu_{k}<\min \left\{c \nu_{1}, \nu_{2}\right\}=\nu_{2} \text { for all } k \neq 1,2 .
$$

Therefore, the event (5) is divided into $K(K-1)$ events

$$
\nu_{k_{1}} \leq \nu_{k_{2}} \leq c \nu_{k_{1}}, \nu_{k_{1}} \leq \nu_{l} \leq \nu_{k_{2}} \text { for } l \neq k_{1}, k_{2}
$$

Here $k_{1}, k_{2}$ are chosen arbitrarily from $1,2, \ldots, K$. Letting

$$
v_{k}=\frac{\left(n_{k}-1\right) \nu_{k}^{2}}{\sigma^{2}} \text { for } k=1,2, \ldots, K
$$

where $\sigma^{2}=\sigma_{1}^{2}=\sigma_{2}^{2}=\cdots=\sigma_{K}^{2}, v_{k}$ is distributed according to $\chi^{2}$ distribution with degrees of freedom $n_{k}-1$. (7) is equivalent to

$$
\lambda_{k_{1}, k_{2}} v_{k_{1}} \leq v_{k_{2}} \leq c \lambda_{k_{1}, k_{2}} v_{k_{1}}, \lambda_{k_{1}, l} v_{k_{1}} \leq v_{l} \leq \lambda_{k_{2}, l} v_{k_{2}} \text { for } l \neq k_{1}, k_{2}
$$

Therefore, we obtain

$$
\begin{gathered}
P\left(c^{-1}<F_{k, l}<c \text { for each pair }(k, l) \text { satisfying } 1 \leq k<l \leq K\right) \\
=\sum_{k_{1}, k_{2}} \int_{0}^{\infty} \int_{\lambda_{k_{1}, k_{2}} x_{1}}^{c \lambda_{k_{1}, k_{2}} x_{1}}\left\{\prod_{l \neq k_{1}, k_{2}} \int_{\lambda_{k_{1}, l} x_{1}}^{\lambda_{k_{2}, l} x_{2}} g_{l}(x) d x\right\} g_{k_{2}}\left(x_{2}\right) d x_{2} g_{k_{1}}\left(x_{1}\right) d x_{1} .
\end{gathered}
$$

If $n_{1}=n_{2}=\cdots=n_{K}=n$,

$$
\begin{aligned}
& P\left(c^{-1}<F_{k, l}<c \text { for each pair }(k, l) \text { satisfying } 1 \leq k<l \leq K\right) \\
& =K(K-1) \int_{0}^{\infty} \int_{x_{1}}^{c x_{1}}\left\{\int_{v_{1}}^{x_{2}} g(x) d x\right\}^{K-2} g\left(x_{2}\right) d x_{2} g\left(x_{1}\right) d x_{1}
\end{aligned}
$$

where $g(x)$ denotes the probability density function of $\chi^{2}$ distribution with degrees of freedom $n-1$.

\section{Numerical examples}

We discussed how to determine the critical value for pairwise comparison satisfying a specified significance level exactly in Section 2. In this section we compare the exact critical value with the conservative critical values determined by Bonferroni's inequality and the improved Bonferroni's inequality through numerical examples regarding the critical values and the power of the test.

Let $K=5$. We set up two types of sample sizes

Sam.1 : (20, 20, 20, 20, 20), Sam.2 : (15, 25, 20, 25, 15).

Let $\alpha=0.05$. Table 1 gives exact critical values and conservative critical values determined by Bonferroni's inequality and the improved Bonferroni's inequality. They are 
the upper critical values. Here E means exact critical values. B and I-B mean conservative critical values determined by Bonferroni's inequality and the improved Bonferroni's inequality respectively. Table 2 gives Type I error using the conservative critical values given in Table 1 . The results of Table 2 were obtained by Monte Carlo simulation with $10,000,000$ times of experiments.

Table 1: Critical values

\begin{tabular}{c|ccc}
\hline & B & I-B & E \\
\hline Sam.1 & 3.862 & 3.831 & 3.659 \\
Sam.2 & 4.163 & 4.131 & 3.881 \\
\hline
\end{tabular}

Table 2: Type I error using the conservative critical value of Table 1

\begin{tabular}{l|cc}
\hline & B & I-B \\
\hline Sam.1 & 0.0371 & 0.0388 \\
Sam.2 & 0.0353 & 0.0369 \\
\hline
\end{tabular}
as follows.

Next, we consider the power of the test. We set up four types of $\left(\sigma_{1}^{2}, \sigma_{2}^{2}, \sigma_{3}^{2}, \sigma_{4}^{2}, \sigma_{5}^{2}\right)$

Case 1. $\sigma_{1}^{2}=\sigma_{3}^{2}=\sigma_{4}^{2}=\sigma_{5}^{2}=1, \sigma_{2}^{2}=\gamma$,

Case 2. $\sigma_{1}^{2}=\sigma_{4}^{2}=\sigma_{5}^{2}=1, \sigma_{2}^{2}=\sigma_{3}^{2}=\gamma$,

Case 3. $\sigma_{1}^{2}=\sigma_{4}^{2}=\sigma_{5}^{2}=1, \sigma_{2}^{2}=\gamma, \sigma_{3}^{2}=\gamma^{2}$,

Case 4. $\sigma_{1}^{2}=\sigma_{5}^{2}=1, \sigma_{2}^{2}=\gamma, \sigma_{3}^{2}=\gamma^{2}, \sigma_{4}^{2}=\gamma^{3}$.

Herein $\gamma=0.4,0.5$. We focus on the all pairs power defined by Ramsey (1978). In Case 1 the power is the probability that $H_{12}, H_{23}, H_{24}, H_{25}$ are rejected. In Case 2 the power is the probability that $H_{12}, H_{13}, H_{24}, H_{25}, H_{34}, H_{35}$ are rejected. In Case 3 the power is the probability that $H_{12}, H_{13}, H_{23}, H_{24}, H_{25}, H_{34}, H_{35}$ are rejected. In Case 4 the power is the probability that $\mathrm{H}_{12}, \mathrm{H}_{13}, \mathrm{H}_{14}, \mathrm{H}_{23}, \mathrm{H}_{24}, \mathrm{H}_{25}, \mathrm{H}_{34}, \mathrm{H}_{35}, \mathrm{H}_{45}$ are rejected. Tables 3 to 6 give the power of Cases 1 to 4 . The results of Tables 3 to 6 were obtained by Monte Carlo simulation with $1,000,000$ times of experiments. The power when $\gamma=0.4$ is uniformly higher than that when $\gamma=0.5$. The power decreases as the number of hypotheses which should be rejected increases.

Table 3: Power in Case 1

\begin{tabular}{c|ccc|ccc}
\hline$\gamma$ & & 0.4 & & & 0.5 & \\
& $\mathrm{~B}$ & $\mathrm{I}-\mathrm{B}$ & $\mathrm{E}$ & $\mathrm{B}$ & $\mathrm{I}-\mathrm{B}$ & $\mathrm{E}$ \\
\hline Sam. 1 & 0.620 & 0.627 & 0.668 & 0.224 & 0.229 & 0.264 \\
Sam. 2 & 0.559 & 0.565 & 0.618 & 0.212 & 0.216 & 0.257 \\
\hline
\end{tabular}

Table 4: Power in Case 2

\begin{tabular}{c|ccc|ccc}
\hline$\gamma$ & & 0.4 & & & 0.5 & \\
& $\mathrm{~B}$ & $\mathrm{I}-\mathrm{B}$ & $\mathrm{E}$ & $\mathrm{B}$ & $\mathrm{I}-\mathrm{B}$ & $\mathrm{E}$ \\
\hline Sam. 1 & 0.519 & 0.527 & 0.576 & 0.118 & 0.123 & 0.151 \\
Sam. 2 & 0.429 & 0.437 & 0.503 & 0.081 & 0.085 & 0.115 \\
\hline
\end{tabular}

Table 5: Power in Case 3 


\begin{tabular}{c|ccc|ccc}
\hline$\gamma$ & \multicolumn{3}{|c|}{0.4} & & 0.5 & \\
& B & I-B & E & B & I-B & E \\
\hline Sam. 1 & 0.534 & 0.544 & 0.598 & 0.066 & 0.071 & 0.097 \\
Sam. 2 & 0.446 & 0.456 & 0.534 & 0.043 & 0.046 & 0.073 \\
\hline
\end{tabular}

Table 6: Power in Case 4

\begin{tabular}{c|ccc|ccc}
\hline$\gamma$ & & 0.4 & & & 0.5 & \\
& $\mathrm{~B}$ & $\mathrm{I}-\mathrm{B}$ & $\mathrm{E}$ & $\mathrm{B}$ & $\mathrm{I}-\mathrm{B}$ & $\mathrm{E}$ \\
\hline Sam. 1 & 0.494 & 0.505 & 0.567 & 0.026 & 0.029 & 0.048 \\
Sam. 2 & 0.394 & 0.405 & 0.493 & 0.011 & 0.013 & 0.028 \\
\hline
\end{tabular}

\section{Conclusions}

In this study we discussed the all-pairwise multiple comparison procedures for checking differences among normal variances. Specifically, we derived the formula for determining the critical value for pairwise comparison satisfying a specified significance level exactly. Then we gave some numerical results regarding critical values and power of the test intended to compare the exact critical value and conservative critical values. Although the test using the exact critical value is more powerful compared to those using the conservative critical values for the single step procedure, we should discuss the multi-step procedures for obtaining higher power. Specifically, we should construct the closed testing procedure referring to Marcus et al. (1976) and construct the sequentially rejective step down procedure referring to Shaffer (1986) and Holland and Copenhaver (1987). We should compare them through numerical examples regarding the power of the test.

\section{Acknowledgement}

The author is deeply grateful to the referee and the editors for their valuable comments and suggestions.

\section{References}

Dunnett, C. W. (1955). A multiple comparison procedure for comparing several treatments with a control. Journal of the American Statistical Association, 50, 1096-1121.

Holland, B. S. and Copenhaver, M. D. (1987). An improved sequentially rejective Bonferroni test procedure. Biometrics, 43 No. 2, 417-423.

Imada, T. (2018). Multiple comparison for checking differences among normal variances. Communications in Statistics-Theory and Method, 47, Issue 18, 4402-4414.

Marcus, R., Peritz, E. and Gabriel, K. R. (1976). On closed testing procedures with special reference to ordered analysis of variance. Biometrika, 63 (3), 655-660.

Ramsey, P. H. (1978). Power differences between pairwise multiple comparisons. Journal of the American Statistical Association, 73, 479-485.

Shaffer, J. P. (1986). Modified sequentially rejective multiple test procedures. Journal of the American Statistical Association, 81, 826- 831. 
Tukey, J. W. (1953). The problem of multiple comparisons. Unpublished manuscript, Princeton University.

Received March 12, 2018

Revised August 29, 2018 